
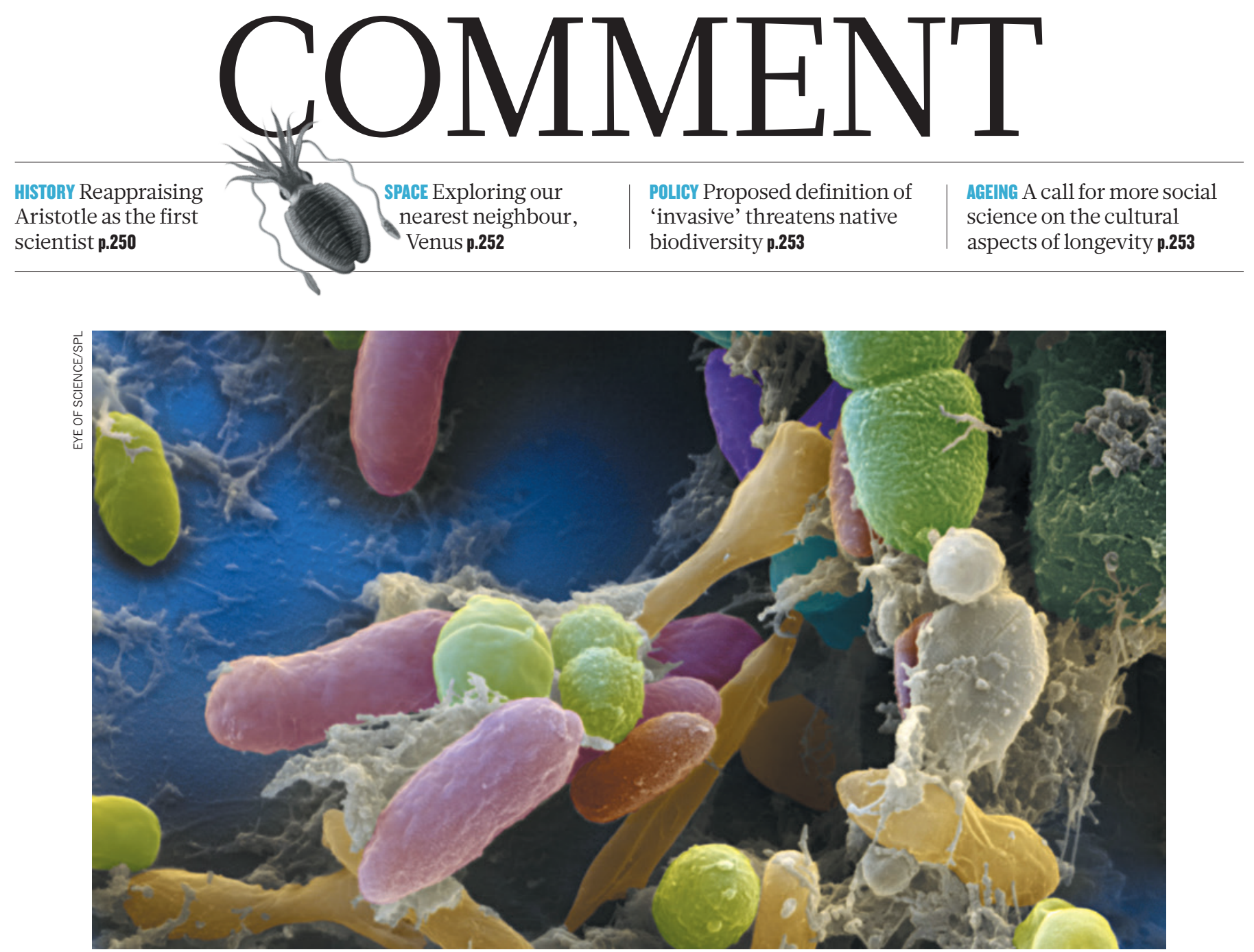

A scanning electron micrograph of bacteria in human faeces, in which $50 \%$ of species originate from the gut.

\title{
Microbiome science needs a healthy dose of scepticism
}

To guard against hype, those interpreting research on the body's microscopic communities should ask five questions, says William P. Hanage.

$\mathrm{E}$ xplorations of how the microscopic communities that inhabit the human body might contribute to health or disease have moved from obscure to ubiquitous. Over the past five years, studies have linked our microbial settlers to conditions as diverse as autism, cancer and diabetes.

This excitement has infected the public imagination. 'We Are Our Bacteria', proclaimed one headline in The New York Times. Some scientists have asserted that antibiotics are causing a great 'extinction' of the microbiome, with dire consequences for human health ${ }^{1}$. Companies offer personalized analysis of the microbial content of faecal samples, promising consumers enlightening information. Separate analyses from the same person can, however, vary considerably, even from the same stool sample. Faecal transplants have been proposed - some more sensible than others - for conditions ranging from diabetes to Alzheimer's disease. With how-to instructions proliferating online, desperate patients must be warned not to attempt these risky procedures on themselves.

Microbiomics risks being drowned in a tsunami of its own hype. Jonathan Eisen, a microbiologist and blogger at the University of California, Davis, bestows awards for "overselling the microbiome"; he finds no shortage of worthy candidates.

Previous 'omics' fields have faltered after murky work slowed progress ${ }^{2}$. Technological advances that allowed researchers to catalogue proteins, metabolites, genetic variants and gene activity led to a spate of associations between molecular states and health conditions. But painstaking further work dampened early excitement. Most initial connections were found to be spurious or, at best, more complicated than originally believed.

The history of science is replete with 
> examples of exciting new fields that promised a gold rush of medicines and health insights but required scepticism and years of slogging to deliver even partially. As such, the criteria for robust microbiome science are instructive for all researchers. As excitement over the microbiome has filtered beyond academic circles, the potential mischief wrought by misunderstanding encompasses journalists, funding bodies and the public.

\section{CRUCIAL QUESTIONS}

Here are five questions that anyone conducting or evaluating this research should ask to keep from getting carried away by hype.

Can experiments detect differences that matter? Profiling a microbiome could produce a catalogue at the level of phyla, species or genes. Much work relies on analysis of $16 S$ rRNA, an ancient gene that tolerates little variation and so is reliably found across the bacterial kingdom. But this allows only a coarse sorting. For example, microbiomes associated with obesity have been distinguished by different ratios of bacterial phyla, which encompass a staggering range of diversity. If this criterion were used to characterize animal communities, an aviary of 100 birds and 25 snails would be considered identical to an aquarium with 8 fish and 2 squid, because each has four times as many vertebrates as molluscs. Even within a single species, strains often differ greatly in the genes they contain.

Modern technology now allows for finer distinctions: we can study more genes in a sample, an ability that may enable us to decipher 'metabolic networks' revealing the biochemical reactions that a microbiome can perform. This kind of analysis could identify gene combinations, potentially from multiple species across a microbial community, that affect health for good or ill. However, pinning an outcome to any particular entity is likely to be hard unless the networks are already well characterized.

To take a simple example from a single bacterial species, we could show that vaccination eliminated $30 \%$ of known pneumococcal strains in a human population - but only because we knew in advance to focus on the genes targeted by the vaccine ${ }^{3}$. Our ability to identify functional differences in closely related genes is rarely sophisticated enough to pull out important genes or networks if we do not know what to look for in the first place. Moreover, genomes are littered with clues both true and false, such as 'hypothetical proteins' and genes that are understood poorly or not at all, but could make for important differences in what metabolic networks do.

We need to be able to identify functional differences in closely related genes from sequence alone. Until then, we must remember that apparent similarities might cloak important differences.
Does the study show causation or just correlation? A separate question is raised when distinct microbiomes can be identified and associated with diseases or other conditions. Then we are left with the chestnut of causes and correlates. Sometimes, a particular microbiome found in association with disease will be merely a bystander ${ }^{4}$.

A 2012 article comparing the gut microbiomes of old people living in care homes with those of old people living in the community found distinct microbiomes that correlated with multiple scores of frailty ${ }^{5}$. After accounting for some potentially confounding factors, the "Press officers authors proposed muststop a causal relationexaggerating ship: diet altered the results, and microbiome, which journalists in turn altered health. muststop This explanation swallowing fits the data, but the them whole." reverse causality the potential for poor health to alter the gut microbiome - was not explored. Frailer people probably have less active immune systems and differences in digestion (such as the time required for food to pass through the stomach and intestines) - factors that could change the microbiome. This work is not the only example of this sort of confusion.

What is the mechanism? All scientists are taught the catechism that correlation is not causation, but correlation almost always implies some sort of causal relationship. We just don't know what it is. We must determine it with careful experiments.

In the past three or four years, studies have advanced from characterizing a broad community of mainly unculturable microbes to identifying functional elements, individual taxa or particular properties. We can now design experiments to precisely define actions of components of the microbiome ${ }^{6}$, for example by reconstituting communities but leaving out specific taxa, or by precisely measuring the biochemical activity of an experimental microbiome in an 'organ on a chip ${ }^{7}$. A return to a reductionist approach is essential if we are to pinpoint both whether the microbiome affects human health, and exactly how it does so.

How much do experiments reflect reality? Even if the microbiome can have an experimental effect, it may not be an important cause of the symptoms seen in ill people.

Much work has addressed the role that gut flora have in obesity, and several studies have found associations between the gut microbiome and weight gain ${ }^{8}$. To assess whether this association was cause or consequence, researchers collected gut-microbiome samples from human twins (one obese, one not) and introduced the microbiota to mice. Mice previously colonized with an 'obese' microbiome lost weight when supplied with a lean microbiome', but only if also fed a normal or low-fat diet. Diet alone had little effect ${ }^{9}$. Although this elegantly controlled experiment suggests great potential for the microbiome and related therapies to affect health, it also shows the microbiome's limits: the effect was dependent on other factors, in this case diet.

Microbiome studies often rely on germfree mice. These animals allow researchers to readily introduce an experimental microbiota. But they do not represent the animals' natural state and are typically unhealthy owing to the lack of a microbiome. So results may not predict responses in animals with flourishing microbiomes. Mice and their microbiomes are also adapted to a rather different niche from humans, so results may not be generalizable.

Could anything else explain the results? There are good reasons to think that bacteria influence us in a host of ways. But there are many other - possibly more important influences, such as diet in the earlier example. Whenever a study links a microbiome to a disease, wise critics should ask whether other contributors to disease are considered, compared and reported.

The hype surrounding microbiome research is dangerous, for individuals who might make ill-informed decisions, and for the scientific enterprise, which needs to develop better experimental methods to generate hypotheses and evaluate conclusions. Funding agencies must not let their priorities be distorted by the buzz around the field, but look dispassionately at the data. Press officers must stop exaggerating results, and journalists must stop swallowing them whole. In pre-scientific times when something happened that people did not understand, they blamed it on spirits. We must resist the urge to transform our microbial passengers into modern-day phantoms.

William P. Hanage is associate professor of epidemiology at the Harvard School of Public Health in Boston, Massachusetts, USA. e-mail:whanage@hsph.harvard.edu

1. Blaser, M. J. Missing Microbes: How the Overuse of Antibiotics is Fueling our Modern Plagues (Henry Holt and Co., 2014)

2. Wilkins, M. R. et al. Proteomics 6, 4-8 (2006).

3. Croucher, N. J. et al. Nature Genet. 45, 656-663 (2013).

4. Shanahan, F. Nutr. Rev. 70 (suppl. 1), S31-S37 (2012).

5. Claesson, M. J. et al. Nature 488, 178-184 (2012)

6. Goodman, A. L. et al. Proc. Natl Acad. Sci. USA 108, 6252-6257 (2011).

7. Huh, D. et al. Nature Protocols 8, 2135-2157 (2013).

8. Ley, R. E. Curr. Opin. Gastroenterol. 26, 5-11 (2010).

9. Ridaura, V. K. et al. Science 341, 1241214 (2013). 